\title{
Fingolimod Exerts only Temporary Antiepileptogenic Effects but Longer-Lasting Positive Effects on Behavior in the WAG/Rij Rat Absence Epilepsy Model
}

\author{
Antonio Leo $^{1}$ - Rita Citraro ${ }^{1}$ Nicola Amodio ${ }^{2}$ - Caterina De Sarro ${ }^{1}$. \\ Maria Eugenia Gallo Cantafio ${ }^{2}$ - Andrew Constanti ${ }^{3}$. Giovambattista De Sarro ${ }^{1}$. \\ Emilio Russo ${ }^{1}$ (1)
}

Published online: 26 June 2017

(C) The American Society for Experimental NeuroTherapeutics, Inc. 2017

\begin{abstract}
One of the major challenges in the epilepsy field is identifying disease-modifying drugs in order to prevent or delay spontaneous recurrent seizure onset or to cure already established epilepsy. It has been recently reported that fingolimod, currently approved for the treatment of relapsing-remitting multiple sclerosis, has demonstrated antiepileptogenic effects in 2 different preclinical models of acquired epilepsy. However, to date, no data exist regarding the role of fingolimod against genetic epilepsy. Therefore, we have addressed this issue by studying the effects of fingolimod in Wistar Albino Glaxo/Rijswijk (WAG/Rij) rats, a wellestablished genetic model of absence epilepsy, epileptogenesis, and neuropsychiatric comorbidity. Our results have demonstrated that an early long-term treatment with fingolimod ( $1 \mathrm{mg} / \mathrm{kg} /$ day $)$, started before absence seizure onset, has both antiepileptogenic and antidepressant-like effects in WAG/Rij rats. However, these effects were transitory, as 5 months after treatment discontinuation, both absence seizure and depressive like-behavior returned to control levels. Furthermore, a temporary reduction of mTOR signaling pathway activity, indicated by reduced phosphorylated mammalian target of rapamycin and phosphorylated p70S6k levels, and
\end{abstract}

Emilio Russo

erusso@unicz.it

1 Science of Health Department, School of Medicine, University "Magna Graecia" of Catanzaro, Catanzaro, Italy

2 Department of Experimental and Clinical Medicine, Magna Graecia University and Translational Medical Oncology Unit, Salvatore Venuta University Campus, Catanzaro, Italy

3 Department of Pharmacology, UCL School of Pharmacy, London, UK by increased phosphorylated Akt in WAG/Rij rats of 6 months of age accompanied the transitory antiepileptogenic effects of fingolimod. Surprisingly, fingolimod has demonstrated longer-lasting positive effects on cognitive decline in this strain. This effect was accompanied by an increased acetylation of lysine 8 of histone $\mathrm{H} 4$ (at both 6 and 10 months of age). In conclusion, our results support the antiepileptogenic effects of fingolimod. However, the antiepileptogenic effects were transitory. Moreover, fingolimod might also have a positive impact on animal behavior and particularly in protecting the development of memory decline.

Keywords Fingolimod $\cdot$ Epileptogenesis $\cdot$ Absence epilepsy $\cdot$ Behavior $\cdot \mathrm{mTOR} \cdot$ Histone deacetylase (HDAC)

\section{Introduction}

Despite the presence of many pressing needs in the epilepsy field, one of the major challenges for modern neurology is identifying disease-modifying drugs. In fact, to date, none of the available antiepileptic drugs has demonstrated clinical efficacy in preventing or delaying spontaneous recurrent seizure onset or in curing already established epilepsy, or even in preventing the burden of neuropsychiatric comorbidities, including cognitive impairment and mood disorders, which represent a primary outcome measure for novel antiepileptic drugs $[1,2]$. Therefore, a good antiepileptogenic treatment should not only counteract seizure onset and/or their course, but it should also improve comorbidities related to epilepsy [3, 4]. "Repurposing" drugs already approved for other diseases could, however, lead to new insights into the epileptogenic process [1]. Accordingly, different commercially available drugs such as those acting on immune and inflammatory 
mechanisms have been tested in different preclinical models of epilepsy [5-7]. Increasing knowledge suggests that the immune system and inflammation are involved in the pathogenesis of epilepsy, thereby representing potentially suitable targets to develop novel disease-modifying drugs [8]. However, the clinical efficacy of anti-inflammatory and immunosuppressant drugs in epilepsy remains to be fully defined [9-11].

Fingolimod (FTY720), an immunomodulating drug derived from the fungus Isaria sinclairii, is the first orally bioavailable disease-modifying drug approved by the US Food and Drug Administration (in September 2010) as a first-line treatment for relapsing-remitting multiple sclerosis $[12,13]$. Chemically, it is a synthetic homologous of sphingosine, derived from membrane lipid sphingomyelin, which, after its phosphorylation into sphingosine 1-phosphate (S1P) by sphingosine kinases, plays a fundamental role in several physiological and pathological functions linked to the immunological, cardiovascular, and central nervous systems [14]. To date, the exact mechanism by which fingolimod acts is not yet completely understood. However, being a prodrug, fingolimod (as well as sphingosine), is phosphorylated in vivo, by sphingosine kinases, into the active metabolite fingolimod phosphate (fingolimod-P or FTY720-P), which acts as a S1P receptor modulator [12, 13, 15]. Furthermore, fingolimod also has receptor-independent effects, some of which are mediated by binding to intracellular targets of S1P [16], including the mammalian target of rapamycin (mTOR) signaling pathway [17-19] and histone deacetylases (HDACs) [20, 21], whereas other effects could be linked to its ability to affect the metabolism and signaling of other lipids [16].

Therefore, these multiple relevant effects have prompted the study of fingolimod effects in other brain disorders [16], including Alzheimer's disease [22] and epilepsy [13, 23, 24]. Regarding epilepsy, Gao et al. [23] described the antiepileptogenic and neuroprotective effects of fingolimod in the lithium-pilocarpine rat epilepsy model through its ability to suppress both microglial activation and to decrease interleukin (IL)-1 $\beta$ and tumor necrosis factor (TNF)- $\alpha$ levels. More recently, the antiepileptic effects of fingolimod were confirmed in the pentylenetetrazole (PTZ)-kindling mouse model with pretreatment reducing seizure development, as well as protecting myelin, and post-treatment reducing seizure severity, as well as inducing remyelination in kindled mice [24]. Considering the potential effects of fingolimod in epilepsy/epileptogenesis and the lack of data on genetic epilepsy models and epilepsy comorbidities, we aimed, in the present study, to evaluate the effects of some fingolimod treatments [acute, subchronic, and early long-term treatment (ELTT)] in Wistar Albino Glaxo/Rijswijk (WAG/Rij) rats, a well-established genetic model of absence epilepsy, epileptogenesis, and neuropsychiatric comorbidity (dysthymia and decline in learning and memory performance)
[25-27]. Despite the fact that neuroinflamamtion does not seem to play a major role in this latter model [28]; it has been demonstrated that drugs (e.g., etoricoxib, indomethacin, and rapamycin) acting on inflammation can both reduce absence seizures and their development and, accordingly, increasing neuroinflammation increases absence seizures [11, 29-31]. The role of inflammatory cytokines has been previously studied by Van Luijtelaar et al. [28]; IL-1 $\beta$ and TNF- $\alpha$ administration can both increase absence seizures in this model, whereas their levels were found to be altered in the blood and/or the brain of WAG/Rij rats at various ages with no clear correlation with spike wave discharge (SWD) development. It was therefore concluded that a possible modulatory effect of neuroinflammation is plausible, but TNF- $\alpha$ might not necessarily have a negative impact, as also suggested by some other previous articles in other models [32]. Furthermore, considering the likely role of the mTOR pathway and HDACs in the etiopathogenesis of idiopathic and acquired epilepsy syndromes and the mechanism of action of fingolimod [33-35], we have also explored a potential effect of fingolimod on these targets in this rat strain.

\section{Materials and Methods}

\section{Animals}

All the experiments were carried out in male nonaudiogenic WAG/Rij rats. WAG/Rij rat progenitors, weighing about $60 \mathrm{~g}$ (4 weeks old), were originally purchased from Charles River Laboratories (Calco, Lecco, Italy) and the rats used in these protocols were all obtained from our breeding colony at the University of Catanzaro animal facility, as previously described $[11,36]$. WAG/Rij rats were housed $3 / 4$ per cage and kept under stable environmental conditions, humidity $(60 \pm 5 \%)$, and temperature $\left(21 \pm 2{ }^{\circ} \mathrm{C}\right)$, in a room with a $12 /$ 12-h reversed light/dark cycle (lights on at 20:00). WAG/Rij rats at 27 days of age (P27) were screened, as previously described [37,38], to evaluate their susceptibility to audiogenic stimuli. Afterwards, only rats without audiogenic susceptibility were used in experiments, considering that WAG/Rij rats expressing audiogenic seizures display higher levels of anxiety than nonaudiogenic WAG/Rij rats [26].

Animal care and experimental procedures were conducted in conformity with the international and national law and policies (EU Directive 2010/63/EU for animal experiments, ARRIVE guidelines and the Basel declaration, including the " $3 \mathrm{R}$ " concept). The experimental protocols and the procedures reported herein were approved by the Animal Care Committee of the University of Catanzaro, Italy. All efforts were made to minimize animal suffering and to reduce the number of animals used. 


\section{Experimental Summary}

The aim of these experiments was to evaluate both the potential antiepileptogenic effects of fingolimod in WAG/Rij rats (experiment 1) and its possible acute and/or subchronic effects versus established absence seizures (experiment 2 ) in the same model. This was accompanied by the analysis of fingolimod effects on different behavioral tasks, which were performed to study anxiety and depressive-like behavior, motor performance, and cognitive impairment [39]. Finally, the effects of fingolimod on the mTOR signaling pathway, as well as on the acetylation level on histone $\mathrm{H} 4$, were explored in order to increase our knowledge of its likely mechanism of action. A scheme of the experimental protocols is reported in Figs. 1 and 2.

\section{Experiment 1}

Male WAG/Rij rats $(n=30)$, after an ELTT with fingolimod ( $1 \mathrm{mg} / \mathrm{kg} /$ day; see subsection "ELTT Protocol" for details), were randomly divided into 5 subgroups $(n=6$; Fig. 1$)$ before being experimentally evaluated [39-41]. Identical matched WAG/Rij rat untreated control subgroups were included in the study. The first subgroup of rats underwent electroencephalography (EEG) recordings both at the age of $\sim 6$ and $\sim 10$ months for the quantification of absence seizures and evaluation of drug effects (see subsection "Surgery and EEG Recordings"). The second subgroup was used to study drug effects on anxiety- and depressive-like behavior in $\sim 6$-monthold rats in the elevated plus maze (EPM) and forced swimming test (FST), respectively. The third subgroup of 6-monthold rats was evaluated in passive avoidance (learning and memory) test and rotarod (motor performance) test. The same schedules for subgroups 2 and 3 were applied in the 2 subgroups (4 and 5) of 10-month-old mice in order to evaluate retention of drug effects $\sim 5$ months after the end of fingolimod ELTT (Fig. 1). The brains of rats in subgroups 2 to 5 were sampled at the end of their respective behavioral tests to study drug effects on the mTOR pathway and on the acetylation levels of lysine 8 of histone $\mathrm{H} 4$.

\section{Experiment 2}

Experiment 2 assessed the effects of 2 doses of fingolimod (1 and $3 \mathrm{mg} / \mathrm{kg}$ ), acutely and subchronically (daily dose) administered in WAG/Rij rats with established seizures at the age of $\sim 6$ months (Fig. 2). WAG/Rij rats $(n=90)$ of $\sim 6$ months of age were randomly divided into 5 groups $(n=18)$ and each of these into 3 subgroups $(n=6)$ for the 2 doses of fingolimod and vehicle (Fig. 2). The first group was used to study (EEGs), both the acute (intraperitoneal injection) and the subchronic (7-day oral treatment) effects of fingolimod on established absence seizures. The second and the third groups were used to assess the acute (intraperitoneal) effects of fingolimod on depressive- and anxiety-like behavior, as well as learning/ memory performance, respectively. The fourth and fifth

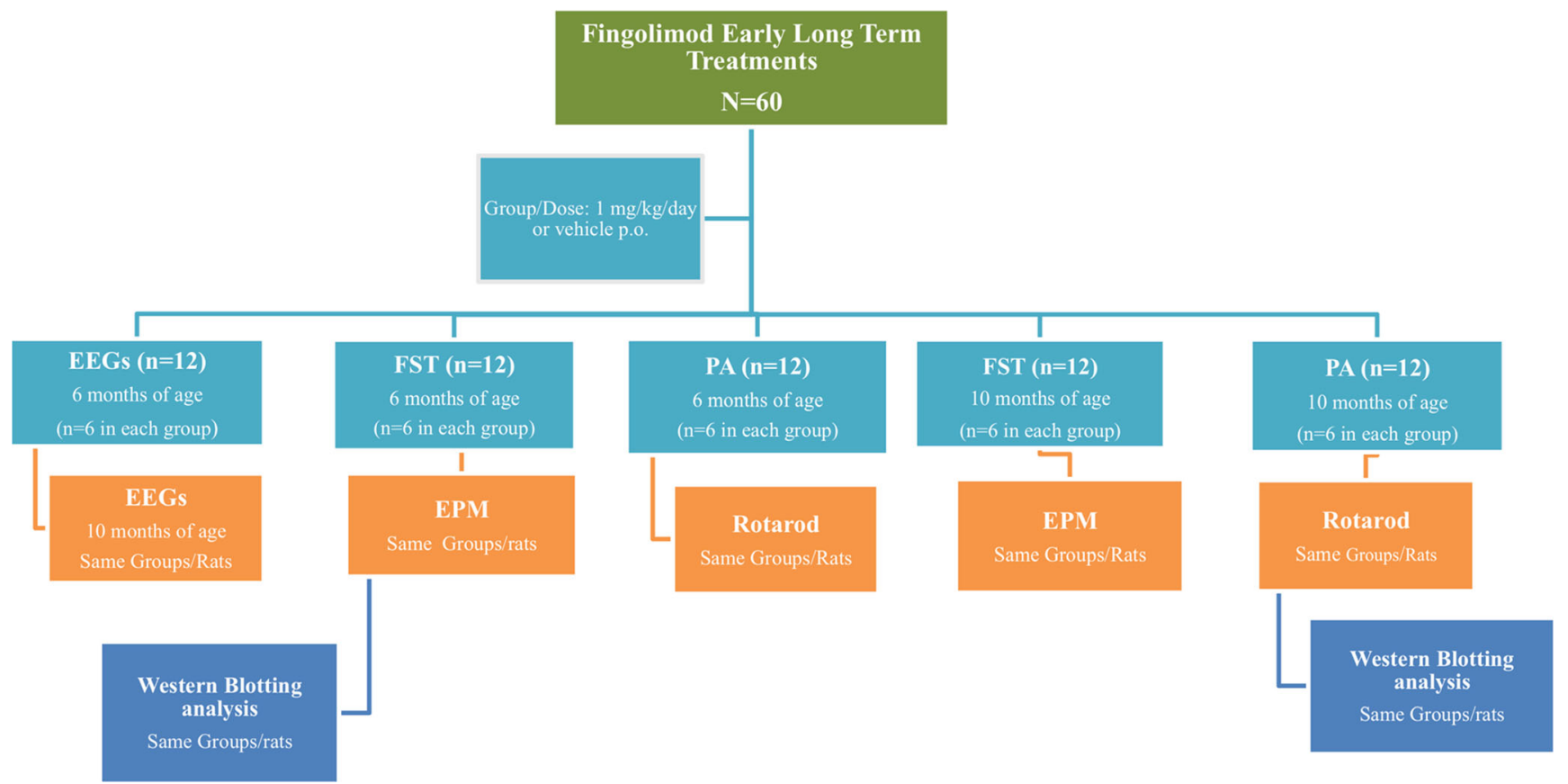

Fig. 1 Experimental protocol 1 for fingolimod early long-term treatment. Graph branches specify the experimental sequence followed and the number of rats used in every test. CTRL = controls; EEGs = electroencephalographic recordings; $\mathrm{EPM}=$ elevated plus maze; FST = forced swimming test; $\mathrm{PA}=$ passive avoidance; i.p. $=$ intraperitoneal administration 


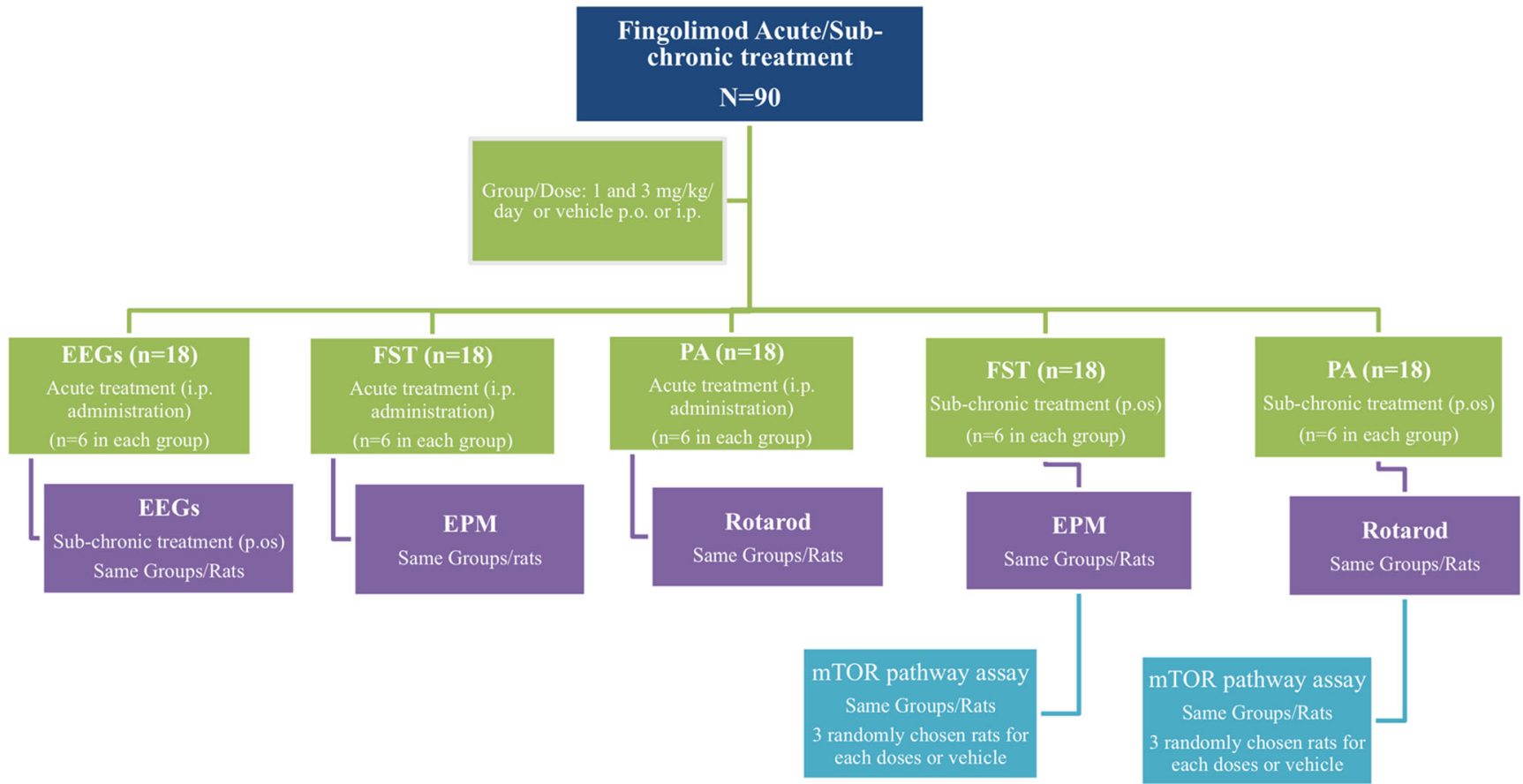

Fig. 2 Experimental protocol 2 for fingolimod acute/subacute treatment. Graph branches specify the experimental sequence followed and the number of rats used in every test. CTRL = controls; EEGs =

groups were used to test subchronic fingolimod effects on the same parameters of groups 2 and 3 (Fig. 2). Six randomly chosen rats from groups 4 and 5 were used to measure the phosphorylation levels of p70S6K in order to study the effects of fingolimod ( $3 \mathrm{mg} / \mathrm{kg} /$ day for 1 week) on the mTOR pathway.

\section{Surgery and EEG Recordings}

WAG/Rij rats allocated into the groups or subgroups for EEG recordings and seizure quantification, under general anesthesia [mixture of tiletamine/zolazepam; 1:1; Zoletil 100®, $50 \mathrm{mg} / \mathrm{kg}$ i.p. (VIRBAC Srl, Milan, Italy)], were stereotaxically implanted with 3 cortical electrodes for EEG recordings attached to a 3-channel rat headmount (8239-SE3; Pinnacle Technology, Stoke-on-Trent, UK), as previously described [11]. After surgery, all animals were allowed at least 1 week of recovery and then connected to preamplifiers [Pinnacle Technology's 8400-9000 video/EEG system with Sirenia Software (Lawrence, KS, USA)] through a flexible recording cable and an electric swivel, attached above the cages, permitting free movements for the animals [39]. Every video-EEG recording was carried out starting at 9:00 for all groups in order to avoid circadian alterations within groups. EEG signals were amplified and conditioned by analog filters (filtering: $<1 \mathrm{~Hz}$ and $>30 \mathrm{~Hz}$ at $6 \mathrm{~dB} /$ octave) and exposed to an analog-to-digital switching with a sampling rate of $300 \mathrm{~Hz}$. The blinded quantification of absence seizures was based on electroencephalographic recordings; EPM elevated plus maze; FST = forced swimming test; $\mathrm{PA}=$ passive avoidance; i.p. = intraperitoneal administration; $\mathrm{mTOR}=$ mammalian target of rapamycin

the number and the duration of EEG SWDs, as previously described $[42,43]$.

\section{Acute and Subchronic Procedures}

Two doses of fingolimod ( 1 and $3 \mathrm{mg} / \mathrm{kg} /$ day; gift from Novartis Pharmaceutical Development, Basel, Switzerland) were used in this protocol section and were chosen according to previous studies $[13,21,23,24]$; the acute effects of fingolimod were always tested $1 \mathrm{~h}$ after intraperitoneal administration considering its known pharmacokinetic profile [44]. However, subchronically treated rats received the drug orally as described for the ELTT, but only for 1 week. All these experiments were run on $\sim 6$-month-old rats $(n=90$ for the entire section) with already established seizures. In order to reduce the number of animal used ( $n=18 v \mathrm{~s}$ 36), rats in the EEG recording group were initially evaluated after acute administration of fingolimod and then treated orally for 1 week and re-evaluated (see subsection "Surgery and EEG Recordings"). All other used rats were divided into groups and subgroups as described above (see subsection "Experimental Summary") and used in behavioral tests.

\section{ELTT Procedure}

WAG/Rij rats $(n=30)$ were administered fingolimod at $1 \mathrm{mg} /$ $\mathrm{kg} /$ day per os starting at $\mathrm{P} 30$ (before seizure onset) up to 
$\sim 5$ months of age ( 17 weeks of treatment). The drug was given in the drinking water by dissolving the desired dose into $120 \mathrm{ml}$ tap water, as previously described [40, 45]. The drug dose was calculated on previously evidence that rats drink $\sim 12 \mathrm{ml} / 100 \mathrm{~g} /$ day; this was subsequently confirmed by checking the volume drunk by rats [40]. Water bottles were wrapped in silver foil to exclude light and solutions were freshly prepared and substituted 3 times a week [41]. After the end of treatment, WAG/Rij rats were normally housed (see subsection "Surgery and EEG Recordings") up to the age of $\sim 6$ months. Age-matched control (vehicle) rats $(n=30)$ were kept under the same housing conditions over the same period of time with vehicle (tap water). During the treatment period, animals were weighed weekly every Monday between 9:00 and 11:00.

At the age of $\sim 6$ months ( 1 month after drug withdrawal), 1 subgroup of treated and 1 of untreated (vehicle) WAG/Rij rats, following surgery (see subsection "Surgery and EEG Recordings"), were experimentally evaluated by $3 \mathrm{~h}$ of EEG recordings over 3 consecutive days. The same rats were studied again via EEG at the age of $\sim 10$ months (5 months after treatment discontinuation) to evaluate the potential long-term effects fingolimod. The same recording schedule was used for subchronically treated rats (see subsection "Acute and Subchronic Procedures" for details on treatment protocol), whereas for the evaluation of potential acute fingolimod effects, rats were subjected to EEG recordings lasting $6 \mathrm{~h}: 1 \mathrm{~h}$ baseline without drug administration, and $5 \mathrm{~h}$ after intraperitoneal injection of fingolimod or vehicle.

\section{Behavioral Tests}

In order to reduce the number of rats used and avoid the influence played by several testings in the same animal, rats ( $n=6$ for every group) were divided as described in Figures 1 and 2 and experimental summary. When 2 tests were performed on the same animal, at least 1 day (range 1-3 days) was allowed as previously described [13, 46, 47]. Experiments were always performed between 09:00 and 11:00 in order to avoid possible circadian alteration of test results. All behavioral tests were performed under controlled environmental conditions, including temperature, humidity, and light intensity (dim illumination), and with the support of video-tracking software (EthoVision XT8; Noldus Information Technology, Wageningen, the Netherlands) [41, 48].

In detail, in order to evaluate the ELTT effects of fingolimod, behavioral tests were performed, respectively, $\sim 1$ and $\sim 5$ months after the end of fingolimod treatment. For the acute and subchronic effects of fingolimod, behavioral tests were performed at the end of the acute and subchronic treatment period ( $1 \mathrm{~h}$ and 7 days, respectively). Regarding subchronic administration groups, when the same group of rats was subjected to multiple behavior tests and/or repeated sessions of the same test, it was kept under treatment. Regarding acute administration groups, the rat groups subjected to the passive avoidance test were injected with fingolimod ( 1 and $3 \mathrm{mg} / \mathrm{kg} /$ day) $1 \mathrm{~h}$ before performing the conditioning session [49], and when multiple tests were required in the same group, the drug was re-injected $1 \mathrm{~h}$ before.

\section{FST}

The FST, despite some limitations [50], is currently used for the experimental study of depressive-like behavior in animals; we used an FST protocol previously standardized in our laboratories [31, 48]. Briefly, rats were placed individually for 6 min into a glass cylinder (height $47 \mathrm{~cm}$, diameter $38 \mathrm{~cm}$ ) filled with $38 \mathrm{~cm}$ water and maintained at $23{ }^{\circ} \mathrm{C}$ to $25^{\circ} \mathrm{C}$. The total duration of immobility (immobility time) was recorded during the last $4 \mathrm{~min}$ of the 6 -min testing period. The criterion for immobility and passive swimming (immobility time) was floating vertically in the water while making only those movements essential to keep the head above the surface of the water, which is directly proportional to depressive-like behavior. At the end of the FST, rats were removed and dried with a towel before being housed. Mean swimming velocity and total distance moved were also measured and examined for every experimental group in order to check for any obvious locomotor impairment [48].

\section{EPM}

The EPM consists of 2 opposing open arms and 2 opposing closed arms of the same size $(45 \mathrm{~cm} \times 10 \mathrm{~cm})$ with walls $10 \mathrm{~cm}$ high connected by a central platform $(10 \times 10 \mathrm{~cm})$ and elevated $80 \mathrm{~cm}$ above the floor, as previously described $[13,47]$. Rats are positioned in the central platform facing a closed arm and the number of entries into, time spent on each arm, and central platform are measured. The maze was systematically cleaned to remove olfactory cues, after each animal was tested. The shorter the time spent in open arms and central platform the higher the anxiety is and vice versa. Mean velocity and total distance moved were also measured and examined for every experimental group $[13,51]$.

\section{Passive Avoidance Test}

In the passive avoidance learning test, used to assess learning and memory, rodents learn to suppress their innate tendencies: moving from the illuminated chamber to the dark chamber $[52,53]$. The passive avoidance-step-through cage (Ugo Basile, Monvalle, Italy), measuring $57 \times 27 \times 30 \mathrm{~cm}$, consists of a cage divided into 2 chambers (light and dark) by a sliding door. The test was conducted over 2 consecutive days as 
previously described [39, 54]. Briefly, during the first day (habituation), rats were placed individually in the light chamber and they were allowed to freely explore the apparatus for 5 min with the sliding door, separating the 2 chambers, open. At the end of this period, rats were returned to their home cage. The conditioning session (learning trial) was started 15 min after habituation. Rats were individually placed in the light chamber. After $30 \mathrm{~s}$ the sliding door was automatically opened. When the rats entered into the dark chamber, the sliding door was automatically closed and an electrical foot shock $(0.5 \mathrm{~mA})$ was delivered for $3 \mathrm{~s}$ via the floor grid. Afterwards, rats were housed. The time taken to enter the dark chamber was recorded and analyzed. Each rat was given $300 \mathrm{~s}$ to enter the dark chamber. If a rat failed to cross from the light to the dark chamber within the cut-off time, it was discarded from the study. Between each training session, the apparatus was systematically cleaned to remove olfactory cues. The retention session (memory trial) was performed $24 \mathrm{~h}$ after the conditioning session by re-introducing the rat into the light chamber of the apparatus. Rats' memory was assessed by recording time taken to enter the dark chamber; however, no foot shock was delivered in this session. The maximum cutoff time for the step-through latency was $300 \mathrm{~s}$. If a rat failed to cross from the light to the dark chamber within the cut-off time, it was housed and a latency of $300 \mathrm{~s}$ was recorded for that rat. Retention memory is directly related to time taken to enter the dark chamber: the better the memory, the greater the latency $[25,39,54]$.

\section{Rotarod Test}

The rotarod test was used to evaluate any eventual locomotor impairment induced by drug treatment. The test was performed as previously described by Monville et al. [55] with some minor modifications, on a Rotarod unit (LE 8500, Panlab, Barcelona, Spain). Briefly, after a habituation session, rats were placed on a rod whose acceleration was increased from 4 to $40 \mathrm{rpm}$ over a period of $300 \mathrm{~s}$. The latency to fall and the number of falls were recorded. Rats were trained for 3 consecutive sessions and the mean of the 3 sessions was analyzed [56].

\section{Western Blotting Analysis}

Rats were decapitated and their brains were quickly removed and submerged in ice-cold artificial cerebrospinal fluid. Subsequently, the cortex was isolated and dissected by an optical microscope as previously described by Russo et al. [29]. The cortex was homogenized using the Gentle MACS dissociator (Miltenyi Biotech, Bergisch Gladbach, Germany) in ice-cold NP40 lysis buffer (Life Technologies) containing a cocktail of protease and phosphatase inhibitors (Life Technologies, Monza, Italy), and then centrifuged at
$16099 \mathrm{~g}$ for $30 \mathrm{~min}$ at $4{ }^{\circ} \mathrm{C}$ to remove tissue debris. Proteins $(50 \mu \mathrm{g})$ were electrophoresed through a NuPAGE $4 \%$ to $12 \%$ gradient gel (Life Technologies) and electroblotted onto a nitrocellulose membrane (Life Technologies) as previously reported [57]. The membrane was blocked for $1 \mathrm{~h}$ with $5 \%$ nonfat dry milk/phosphate-buffered saline-Tween $0.05 \%$ (Biorad, Hercules, CA, USA), and then incubated over night with the antibodies for phosphorylated (p) Akt (p-Akt; S473), AKT, p-mTOR (S2448), mTOR, p-p70S6 kinase (T389), p70S6 kinase, HDAC1, histone $\mathrm{H} 4$, and acetylated-histone H4 (K8) (all from Cell Signaling, Danvers, MA, USA); glyceraldehyde 3-phosphate dehydrogenase (Santa Cruz Biotechnologies, Santa Cruz, CA, USA) was used as loading control. The levels of proteins and phosphoproteins were detected with horseradish peroxidase-linked secondary antibodies and the enhanced chemiluminescence system (GE Healthcare, Milan, Italy). Autoradiographs were scanned to obtain arbitrary densitometric units. Data were normalized against those of the corresponding glyceraldehyde 3phosphate dehydrogenase. The experiments were performed in triplicate and the results calculated as means \pm SEM and expressed as protein change (\%) [57].

\section{Statistical Analysis}

All statistical procedures were performed using GraphPad Prism 6.0 (GraphPad Software, Inc., La Jolla, CA, USA). EEG recordings were subdivided into 30-min epochs, and the duration and number of SWDs were evaluated separately, as previously described [42]. Such values were averaged and data were expressed as means \pm SEM. EEG data were analyzed and compared by 1-way analysis of variance (ANOVA) followed by Dunnett's post-hoc test. Furthermore, we used 1-way ANOVA followed by Dunnett's post-hoc test to analyze and compare behavioral data obtained from acute and subchronic treatments. Data obtained by behavioral tests and Western blotting analysis from the ELTT schedule were analyzed and compared by 2-way ANOVA followed by Bonferroni's post-hoc test. Data obtained by Western blotting analysis from subchronic treatment were analyzed and compared by the Student's $t$-test. All tests used were 2 sided and $p \leq 0.05$ was considered significant.

\section{Results}

\section{Effects of Acute and Subchronic Treatment With Fingolimod on Established Absence Seizures}

Analysis of EEG recordings of 6-month-old WAG/Rij rats showing established absence seizures indicated that fingolimod, administered acutely by intraperitoneal injection and subchronically by the oral route ( 1 and $3 \mathrm{mg} / \mathrm{kg}$ ) was not 
able to significantly modify $(p>0.05)$ absence seizure parameters in comparison with the control group (data not shown).

\section{Effects of ELTT With Fingolimod on Absence Seizure Development}

Untreated 6-month-old control WAG/Rij rats showed a mean number of SWDs (nSWDs) of $11.06 \pm 1.02$, with a mean total duration (dSWDs) of $72.03 \pm 6.22 \mathrm{~s}$ and a mean single duration (sSWD) of $6.31 \pm 1.33$ for a 30 -min epoch (Fig. 3). ELTT with fingolimod ( $1 \mathrm{mg} / \mathrm{kg} /$ day p.o.) significantly decreased $(p<0.05)$ the development of absence seizures (both number and total duration but not sSWD) in WAG/Rij rats at the age of 6 months (1 month after treatment discontinuation) compared with untreated rats of the same age, supporting the hypothesis of antiepileptogenic effects (Fig. 3). In particular, ELTT with fingolimod did not significantly $(\sim 12 \% ; P>0.05)$ reduce mean sSWD duration, whereas it significantly modified nSWD, as well as dSWD by about $30 \%(p=0.003)$ and $40 \%$ ( $p=0.00029$ ), respectively (Fig. 3).

In contrast, in fingolimod-treated rats at 10 months of age (5 months after drug discontinuation), absence seizure parameters (nSWD, dSWD, and sSWD) were no longer significantly $(p>0.05)$ modified in comparison with the respective untreated control group of the same age (Fig. 3). Animal growth, over the 17 weeks of treatment, did not significantly differ between fingolimod-treated and untreated rats (data not shown).

\section{Effects of Fingolimod on Depressive- and Anxiety-Like Behavior}

WAG/Rij rats at the age of 4 months and even more so at 6 months of age display an increased immobility time in the FST, which is very likely linked to the development of SWDs $[26,40]$. Both fingolimod acute and subchronic treatments had no effects on immobility time in any group tested, despite animal age or dose used. However, fingolimod ELTT (1 mg/ $\lg$ /day p.o.) significantly $(p=0.0054)$ decreased the immobility time in 6-month-old WAG/Rij rats (Fig. 4a; 1 month after treatment discontinuation), whereas this fingolimod antidepressant-like effect was not observed in WAG/Rij rats where treatment was discontinued 5 months earlier (10month-old rat group; Fig. 4a). Mean velocity and total distance moved did not significantly differ $(p>0.05)$ between groups (data not shown). Anxiety-like behavior in WAG/Rij rats was evaluated by EPM and was not affected by either acute or subchronic fingolimod treatment. Similarly, ELTT with fingolimod was not able to modify any measured parameters independently of the time of the test (Fig. 4b).

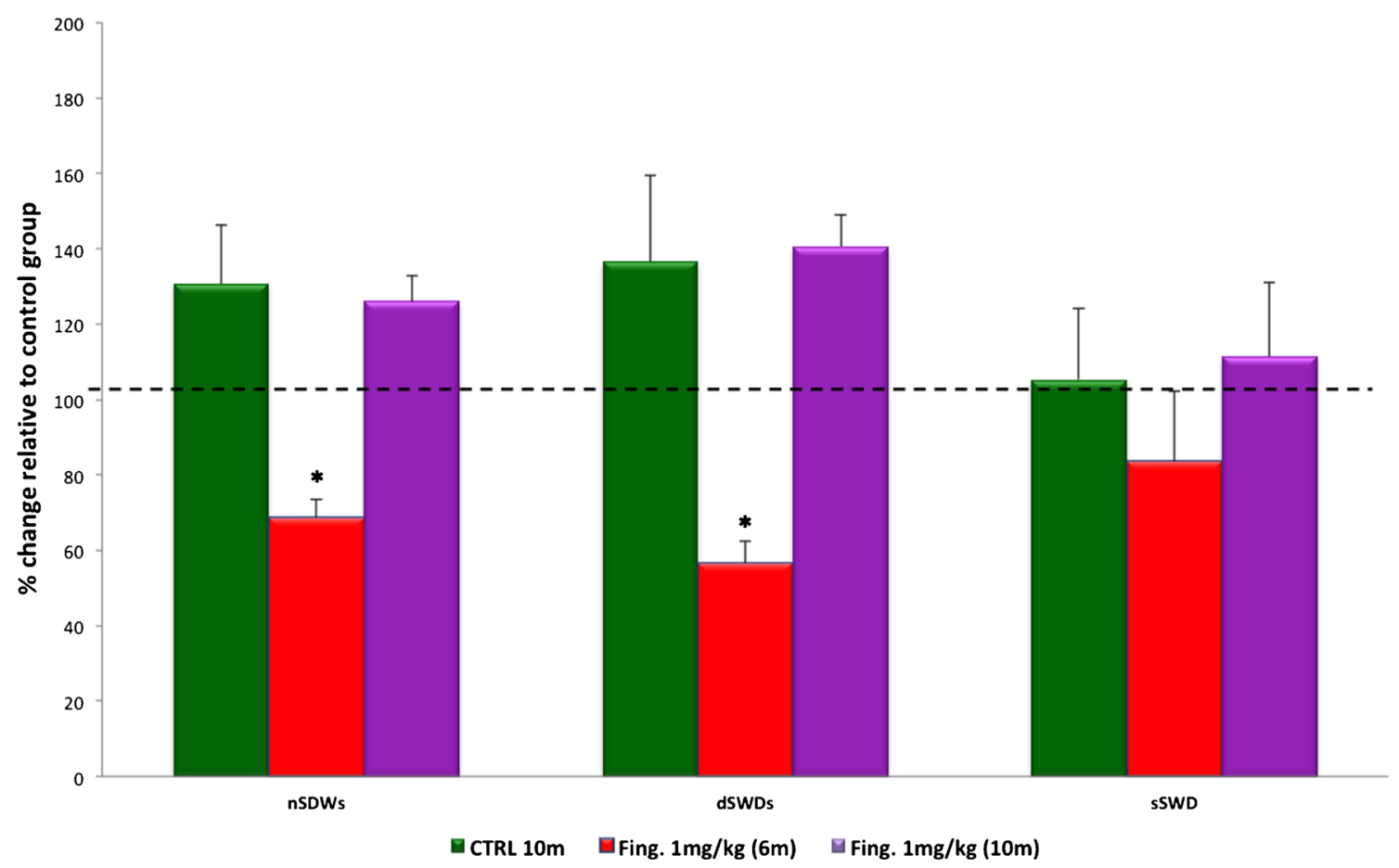

Fig. 3 Effects of an early long-term fingolimod treatment on the development of absence seizures. Effects of an early long-term treatment (starting at P30 and lasting 17 weeks) with fingolimod (Fing.) on spike-wave discharges (SWDs) recorded in Wistar Albino Glaxo/Rijswijk rats at $6(6 \mathrm{~m})$ and $10(10 \mathrm{~m})$ months of age. Data (means $\pm \mathrm{SEM}, n=6$ per group) are expressed as percentage change relative to 6-month-old control rats (dotted line; values for control rats were: $\mathrm{nSWDs}=11.06 \pm 1.02 ; \mathrm{dSWDs}=72.03$ \pm 6.22 ; nSWDs $=6.31 \pm 1.33$ ). CTRL $=$ control rats; nSWDs $=$ mean number of SWDs for every 30-min epoch; dSWDs = mean cumulative duration of SWDs for every 30-min epoch expressed in seconds(s); sSWD = mean duration of a single SWD expressed in (s). *Significantly different $(p<0.05)$ from age-matched control rats 

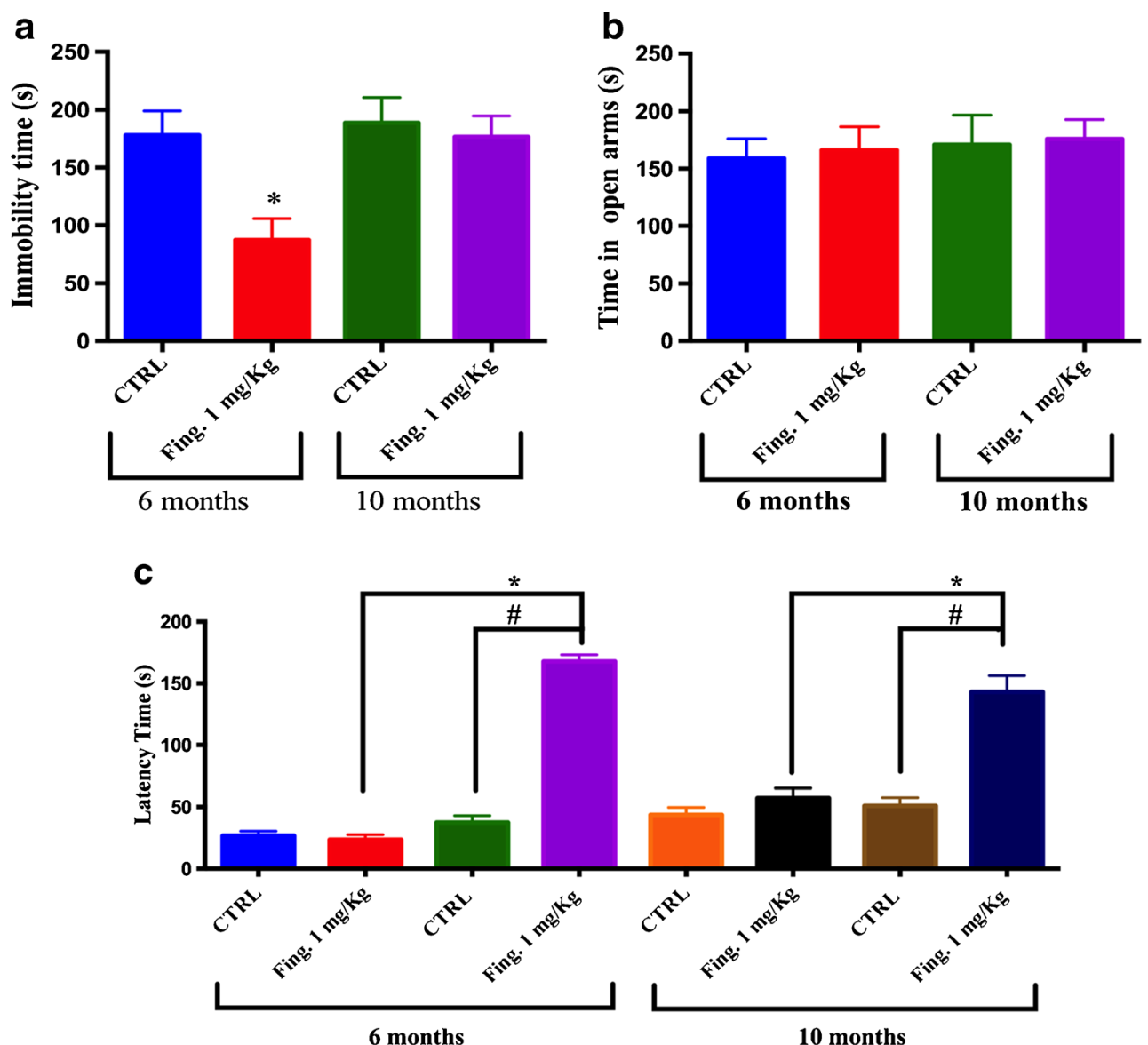

Fig. 4 (a) Forced swimming test (FST). Bars indicate the immobility time, expressed in seconds, in the FST in Wistar Albino Glaxo/Rijswijk (WAG/Rij; $n=6$ per group) rats at 6 and 10 months of age following an early long-term treatment (ELTT; started at P30 and lasting 17 weeks) with fingolimod at $1 \mathrm{mg} / \mathrm{kg} /$ day (Fing.). *Significantly different $(p<0.05)$ from age-matched control rats (CTRL). (b) Elevated plus maze (EPM). Bars indicate the time spent in open arms, expressed in seconds,

\section{Effects of Fingolimod on Learning and Memory and Motor Coordination}

Learning and memory decline has been recently identified in WAG/Rij rats older than 6 months [25, 54]. In agreement with our results in other behavioral tests, acute and subchronic fingolimod treatment did not influence learning and memory evaluated in the passive avoidance test. However, fingolimod ELTT $(1 \mathrm{mg} / \mathrm{kg} /$ day p.o.) significantly $(p=0.0023)$ increased the time taken to enter the dark chamber during the retention session both in 6- and 10-month-old rats, 1 and 5 months after treatment discontinuation (Fig. 4c). Fingolimod did not influence rotarod test results in any group.

\section{Effects of Fingolimod on the mTOR Pathway}

By comparing 6-month-old control rats with 10-month-old rats, we found that mTOR and p70S6K phosphorylation in the EPM in WAG/Rij ( $n=6$ per group) rats at 6 and 10 months of age following an ELTT with fingolimod at $1 \mathrm{mg} / \mathrm{kg} / \mathrm{day}$ (Fing.). *Significantly different $(p<0.05)$ from age-matched control rats (CTRL). (c) Passive avoidance test. Bars indicate the time taken to enter the dark chamber. *Significantly different $(p<0.05)$ from age-matched fingolimod-treated rats; \#Significantly different $(p<0.05)$ from agematched control (untreated) WAG/Rij rats. Data are means \pm SEM

levels were not modified by age, whereas AKT was significantly $(p=0.0047)$ more phosphorylated in older animals (Fig. 5b). The subchronic administration of fingolimod ( $3 \mathrm{mg} / \mathrm{kg} /$ day for 1 week) was not able to reduce the amount of p-p70S6K and the ratio of p-p70S6K:p70S6K in the cortex of 6-month-old WAG/Rij rats (Fig. 6).

Western blotting analysis of the rat cortex obtained from animals in the fingolimod $(1 \mathrm{mg} / \mathrm{kg} /$ day $)$ ELTT group at 6 months of age revealed increased $(p=0.0017)$ phosphorylation of AKT and reduced $(P<0.001)$ phosphorylation of both mTOR and p70S6K. However, no differences were found when comparing phosphorylation levels in 10-monthold rats previously treated with fingolimod (Fig. 5).

\section{Effects of Fingolimod on Histone Acetylation}

No significant $(P>0.05)$ difference in the acetylation of lysine 8 of histone $\mathrm{H} 4$ was observed between untreated 6-month-old 
Fig. 5 Western blot analysis after early long-term fingolimod treatment (ELTT). (a) Representative panel of Western blotting experiments on the effect of an ELTT with fingolimod (Fing.) on the expression level in the cortex, of phosphorylated (p) Akt; (b) phosphorylated mammalian target of rapamycin (p-mTOR); (c) and phosphorylated p70S6K (pp70S6K). Columns represent mean relative protein levels normalized to control ( $n=6$ per group). Loading was normalized using glyceraldehyde 3phosphate dehydrogenase (GADPH) levels. *Significantly different $(p<0.05)$ from agematched control (untreated) Wistar Albino Glaxo/Rijswijk (WAG/Rij) rats; " significantly different $(p<0.05)$ from agematched control (untreated) $\mathrm{WAG} / \mathrm{Rij}$ rats. $\mathrm{CTRL}=$ control a

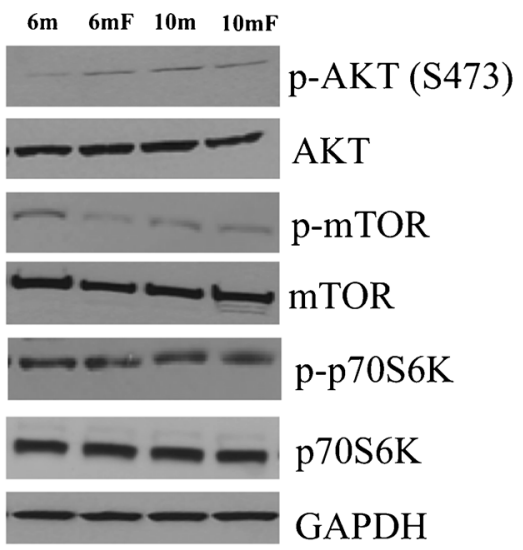

c

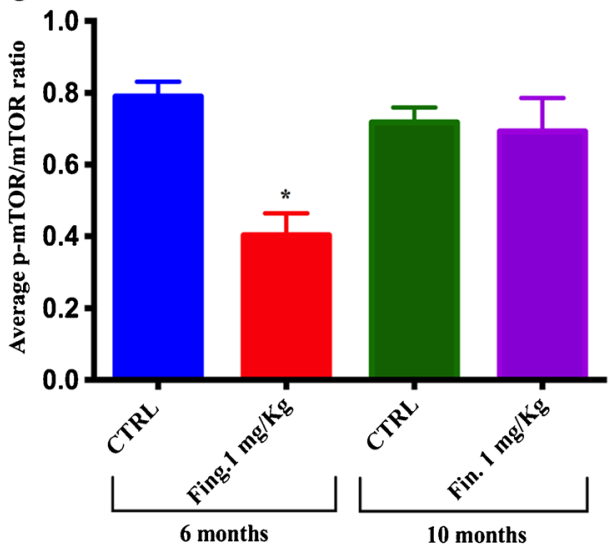

b

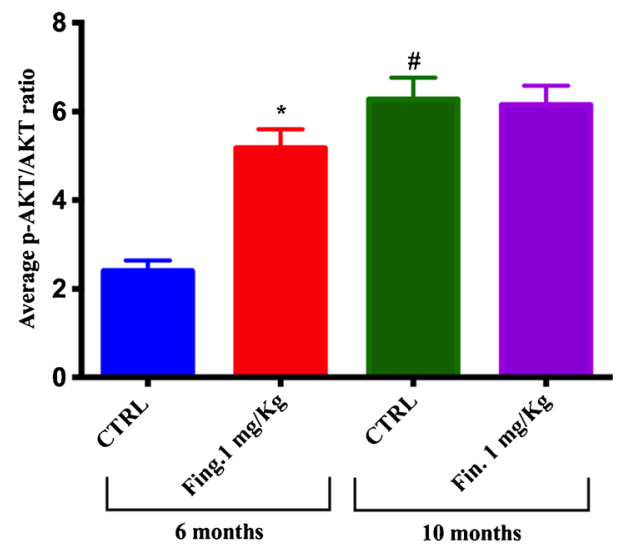

d

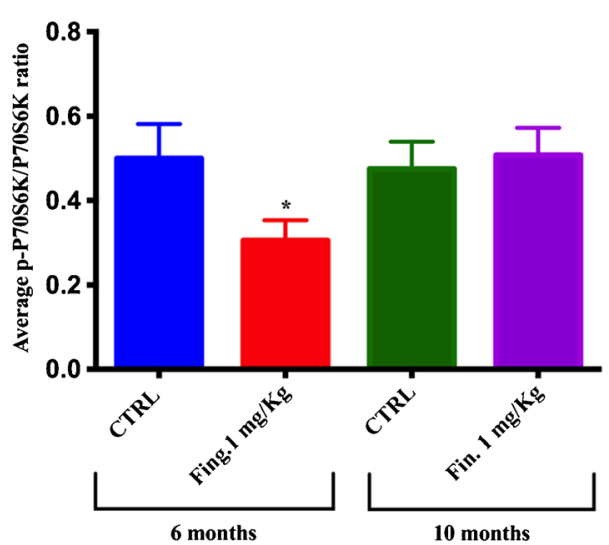

WAG/Rij rats compared with untreated 10-month-old WAG/ Rij rats (Fig. 7b). Fingolimod ELTT significantly increased $(P=0.0009)$ acetylation of lysine 8 of histone $\mathrm{H} 4$ (H4K8) in WAG/Rij rats both at 6 and 10 months of age compared with respective control groups (Fig. 7b). Furthermore, no significant $(P>0.05)$ difference in HDAC1 expression levels was observed after an ELTT with fingolimod (Fig. 7a).
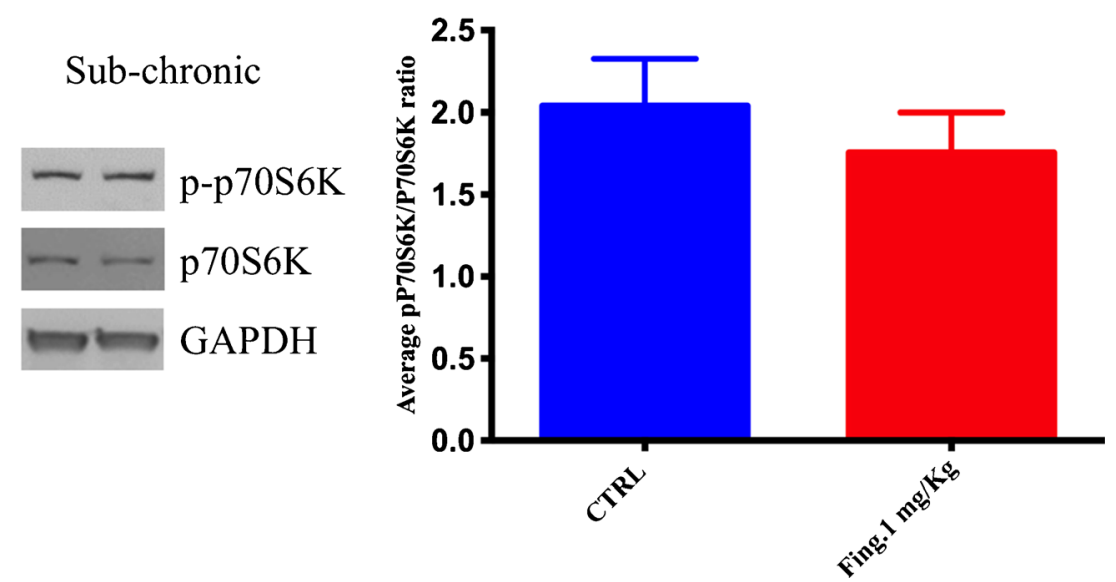

Fig. 6 Western blot analysis after subchronic fingolimod treatment. Quantitative Western blot analysis of phosphorylated p70S6K (p-p70S6K) levels in the cortex of Wistar Albino Glaxo/Rijswijk rats of 6 months of age subchronically treated with fingolimod. Columns represent mean relative protein levels normalized to control ( $n=6$ per group). Loading was normalized using glyceraldehyde 3-phosphate dehydrogenase (GADPH) levels. $\mathrm{CTRL}=$ control 
Fig. 7 Quantitative Western blot analysis of the acetylation of lysine 8 of histone $\mathrm{H} 4$.

*Significantly different $(p<0.05)$ from age-matched control (untreated) Wistar Albino Glaxo/ Rijswijk (WAG/Rij) rats; " significantly different $(p<0.05)$ from age-matched control (untreated) WAG/Rij rats
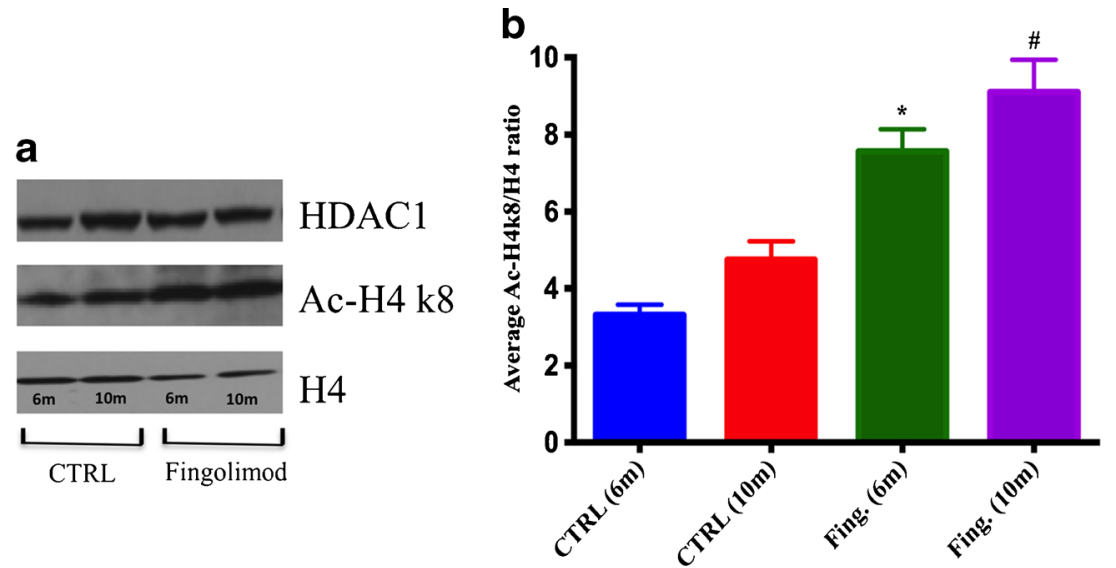

\section{Discussion}

Our results demonstrate that an ELTT (17 weeks) with fingolimod, started before absence seizure onset (treatment started at P30 and seizure onset at about P60), has both antiepileptogenic and antidepressive-like effects in the WAG/Rij rat absence epilepsy model [27]. However, these effects, as reported for the majority of the drugs tested in this model so far, were transitory, as 5 months after treatment discontinuation, both absence seizures and depressive-like behavior (which usually accompanies seizure development in WAG/Rij rats), returned to control level [26, 27, 31]. Furthermore, comparing fingolimod effects in WAG/Rij rats of 6 months of age (i.e., $30 \%$ absence seizure development reduction) with the results obtained for other drugs previously tested, it seems that fingolimod is probably the least effective drug tested so far (compare fingolimod effects $v s$ all other drugs reported in the review by Russo et al. [27]).

The antiepileptogenic effects of fingolimod have also been previously demonstrated in the rat lithium-pilocarpine epilepsy model where a $1 \mathrm{mg} / \mathrm{kg} /$ day treatment for 14 consecutive days started $24 \mathrm{~h}$ after onset of status epilepticus was able to decrease both glial activation and associated abnormal expression of IL- $1 \beta$ and TNF- $\alpha$ which are rapidly overexpressed after status epilepticus. Furthermore, in this latter study, fingolimod administered during the silent phase was able to decrease the development of spontaneous seizures together with a reduction in their duration and severity [23]. These effects could also be the result of astrocyte S1P receptor modulation; however, further studies are needed to better clarify this hypothesis [13, 23]. More recently, Gol et al. [24] also demonstrated that fingolimod, through neuroprotective and anti-inflammatory effects, promotes myelin protection and remyelination improvement in the PTZ-kindling mouse model of epilepsy. In particular, the pretreatment with fingolimod, $1 \mathrm{~h}$ before PTZ administration resulted in decreased seizure onset, microglial activation, and neuronal death in hippocampal areas CA1 and CA3. Likewise, post-treatment with fingolimod decreased seizures and promoted the endogenous remyelination processes in kindled mice. Accordingly, based on this background, it is not possible to exclude the notion that fingolimod can exert antiepileptogenic effects in this strain through some type of antiinflammatory mechanism, which could also be linked to modulation of neuronal S1P receptors [13]. It is known that glial activation and the related overexpression of proinflammatory cytokines seem to play a crucial role in epileptogenesis both in humans and in several animal models of epilepsy [58-61]; however, to date, such a relationship between neuroinflammation and absence seizure development in WAG/Rij rats remains unclear [27, 28]. Indeed, neuroinflammation and related mediators worsen absence seizures in this strain [28-30,62,63], while cyclooxygenase inhibitors have some partial antiabsence properties [11, 63, 64] and etoricoxib, a selective cyclooxygenase 2 inhibitor, also possesses antiepileptogenic effects in this strain, which appear to be more effective than fingolimod with a reduction in the development of absence seizures of about $45 \%$ versus $30 \%$ obtained with fingolimod [11]. Overall, based on the current knowledge, neuroinflammation does not convincingly seem to take part in the epileptogenic process in WAG/Rij rats; rather, it seems to accompany and participate in seizure generation and synchronization [27], as also supported by findings in the GAERS model of absence epilepsy [65]; accordingly, these mechanisms may contribute to the limited fingolimod effects in this model.

To date, several animal models and some clinical studies have demonstrated that the mTOR signaling pathway plays a relevant role in the development of some idiopathic and acquired epilepsy syndromes [33, 35]. Furthermore, the AktmTOR pathway was more active (hyperphosphorylated) in young WAG/Rij rats before the onset of absence seizures, supporting the role of the mTOR pathway as one of the possible causes of the epileptogenic process in WAG/Rij rats [27, 66]. However, absence seizures per se might also activate the mTOR signaling pathway, as also supported by the fact that a single administration of PTZ in rodents increases mTOR 
pathway activity $[66,67]$. As it was known that fingolimod is also able to inhibit the Akt-mTOR pathway both in the experimental autoimmune encephalomyelitis mouse model [17] and in the glioblastoma cell line [19], we investigated its effects on this pathway.

Fingolimod ELTT resulted in a temporary reduction of mTOR signaling pathway activity, indicated by reduced pmTOR and p-p70S6k levels and by increased p-Akt in 6month-old WAG/Rij rats; this modulatory effect accompanied the transitory antiepileptogenic fingolimod effects. In fact, 5 months after treatment discontinuation, the mTOR pathway activation returned to control levels together with the incidence of absence seizures. These results suggest that the inhibitory effects of fingolimod ELTT could be indirect and linked to its antiepileptogenic effects (reduction of absence seizures), which would, in turn, reduce mTOR pathway activation. This hypothesis is also supported by the fact that a subchronic treatment with fingolimod neither reduced mTOR pathway activation nor absence seizures in adult WAG/Rij rats. Furthermore, it was previously demonstrated that the mTOR inhibitor rapamycin has both antiepileptogenic effects (about 50\% seizure development decrease vs 30\% obtained with fingolimod) and some, albeit limited, antiabsence effects with long-lasting antiepileptogenic effects [29, 31]. Of note, we found an age-dependent increase in the phosphorylation of p-Akt in older control rats [68]; however, the significance of this result still remains controversial [69].

Previous studies have documented the linkage between epilepsy and depression both in preclinical models and in humans [70, 71]. Regarding WAG/Rij rats, it has also been hypothesized that some common, currently unknown, mechanisms could be responsible for the appearance of absence seizures and low-grade depression (dysthymia) in this strain, with seizure activity being required for the expression of depressivelike behavior [26, 72], even though some exceptions exist [27, 40, 41]. Based on this background, the antidepressant effects of fingolimod observed after an ELTT in WAG/Rij rats could be linked to its temporary antiepileptogenic effects, which disappear with seizure reappearance 4 months later, rather than to a direct effect. Acute and subchronic treatments with fingolimod were not associated with a reduction of established absence seizures and immobility time in adult epileptic WAG/Rij rats, thereby supporting this view. However, a direct effect on depressive symptoms might not be excluded. In fact, the antidepressant effects of fingolimod $(3 \mathrm{mg} / \mathrm{kg}$ i.p. once a day for 4 weeks) have been reported in mice exposed to chronic unpredictable stress and in mice chronically treated with corticosterone, both of which represent models of depression [21], and in patients with relapsing-remitting multiple sclerosis who switched from injectable disease-modifying therapy to fingolimod [73, 74]. In our study, fingolimod did not affect anxiety-like behavior in the EPM test, similar to the findings by di Nuzzo et al. [21] in mice.
At odds with these temporary effects, fingolimod ELTT (but not acutely or subchronically) prevented the development of cognitive decline and this effect was maintained up to 5 months after drug suspension. Memory impairment was only recently demonstrated in WAG/Rij rats $[25,54]$. Karson et al. [54] demonstrated that at 5 months of age (range 4-6 months) WAG/Rij rats do not differ from Wistar rats, whereas they have an impairment in learning and memory at 13 months of age (range 12-14 months); however, Jafarian et al. [25] reported that WAG/Rij rats of 6 months of age display memory impairment accompanied by hippocampal neuronal death. Accordingly, we found that there was no difference in WAG/Rij rats aged 6 or 10 months and fingolimod effects were statistically similar at both ages and therefore not temporally related to its antiepileptogenic effects. Despite these 2 studies, it still remains to be clarified whether seizures and learning/memory impairment are independent or related processes; our results support the hypothesis that they are not dependent; however, further studies are needed.

Considering the ability of fingolimod to inhibit HDAC [20] and the role of HDAC inhibitors, including valproate and lacosamide, in enhancing learning and memory processes and the epigenetic modulation of absence seizure development in this model [27, 75-79], we studied the effects of fingolimod ELTT on acetylation of lysine 8 of histone H4 (at both 6 and 10 months of age) and found a significant increase temporally linked to the preserved cognitive functions. As previously reported, this epigenetic regulation by fingolimod could also lead to an augmented expression of growth factors, such as brain-derived neurotrophic factor, that play a fundamental role in synaptic plasticity process, which are involved in memory formation and retention [21, 80-82]. In any case, the role of HDAC modulation in epilepsy and, more specifically, in WAG/Rij rats still does not permit us to either support or discard its involvement in fingolimod effects, also considering the potential of HDAC modulation in epileptogenesis and animal behavior [34, 83, 84].

\section{Conclusions}

Fingolimod and the related S1P signaling have recently gathered attention in epilepsy [13]; furthermore, fingolimod also possesses other mechanisms of action, which might be relevant for this neurological disease. Our results further extend the current knowledge supporting potential antiepileptogenic effects of this drug; however, in our experiments, these effects were very limited in comparison with previous experiments with other drugs in this model and not permanent. Moreover, fingolimod might also have a positive impact on animal behavior and particularly in protecting the development of cognitive decline associated with epilepsy. In conclusion, fingolimod might be considered a promising antiepileptogenic treatment on the basis of the current view of the several unmet 
needs in this field $[4,5,85]$; however, further experiments are needed in order to clarify the exact mechanism(s) by which fingolimod exerts these potentially beneficial effects in this neurological disorder.

Acknowledgements We thank the Novartis Institute for BioMedical Research (NIBR) (Basel, Switzerland) for the kind gift of fingolimod.

Required Author Forms Disclosure forms provided by the authors are available with the online version of this article.

\section{References}

1. Kaminski RM, Rogawski MA, Klitgaard H. The potential of antiseizure drugs and agents that act on novel molecular targets as antiepileptogenic treatments. Neurotherapeutics. 2014;11:385-400.

2. Franco V, French JA, Perucca E. Challenges in the clinical development of new antiepileptic drugs. Pharmacol Res. 2016;103:95104.

3. Terrone G, Pauletti A, Pascente R, Vezzani A. Preventing epileptogenesis: A realistic goal? Pharmacol Res 2016;110:96-100.

4. Brooks-Kayal AR, Bath KG, Berg AT, et al. Issues related to symptomatic and disease-modifying treatments affecting cognitive and neuropsychiatric comorbidities of epilepsy. Epilepsia. 2013;54(Suppl. 4):44-60.

5. White HS, Loscher W. Searching for the ideal antiepileptogenic agent in experimental models: single treatment versus combinatorial treatment strategies. Neurotherapeutics. 2014;11:373-384.

6. Kobow K, Auvin S, Jensen F, et al. Finding a better drug for epilepsy: antiepileptogenesis targets. Epilepsia. 2012;53:1868-1876.

7. Loscher W, Klitgaard H, Twyman RE, Schmidt D. New avenues for anti-epileptic drug discovery and development. Nat Rev Drug Discov. 2013;12:757-776

8. Rajasekaran K, Goodkin HP. A swell in the armamentarium of antiepileptic drug targets. Epilepsy Curr 2011;11:172-176.

9. Beghi E, Shorvon S. Antiepileptic drugs and the immune system. Epilepsia. 2011;52(Suppl. 3):40-44.

10. Marchi N, Granata T, Janigro D. Inflammatory pathways of seizure disorders. Trends Neurosci 2014;37:55-65.

11. Citraro R, Leo A, Marra R, De Sarro G, Russo E. Antiepileptogenic effects of the selective COX-2 inhibitor etoricoxib, on the development of spontaneous absence seizures in WAG/Rij rats. Brain Res Bull 2015;113:1-7.

12. Brinkmann V, Billich A, Baumruker T, et al. Fingolimod (FTY720): discovery and development of an oral drug to treat multiple sclerosis. Nat Rev Drug Discov 2010;9:883-897.

13. Russo E, Leo A, Crupi R, et al. Everolimus improves memory and learning while worsening depressive- and anxiety-like behavior in an animal model of depression. J Psychiatr Res 2016;78:1-10.

14. Rosen H, Stevens RC, Hanson M, Roberts E, Oldstone MB. Sphingosine-1-phosphate and its receptors: structure, signaling, and influence. Annu Rev Biochem 2013;82:637-662.

15. Groves A, Kihara Y, Chun J. Fingolimod: direct CNS effects of sphingosine 1-phosphate (S1P) receptor modulation and implications in multiple sclerosis therapy. J Neurol Sci 2013;328:9-18.

16. Brunkhorst R, Vutukuri R, Pfeilschifter W. Fingolimod for the treatment of neurological diseases-state of play and future perspectives. Front Cell Neurosci 2014;8:283.

17. Hou H, Cao R, Miao J, et al. Fingolimod ameliorates the development of experimental autoimmune encephalomyelitis by inhibiting Akt-mTOR axis in mice. Int Immunopharmacol 2016;30:171-178.
18. Marvaso G, Barone A, Amodio N, et al. Sphingosine analog fingolimod (FTY720) increases radiation sensitivity of human breast cancer cells in vitro. Cancer Biol Ther 2014;15:797-805.

19. Zhang L, Wang H, Zhu J, Ding K, Xu J. FTY720 reduces migration and invasion of human glioblastoma cell lines via inhibiting the $\mathrm{PI} 3 \mathrm{~K} / \mathrm{AKT} / \mathrm{mTOR} / \mathrm{p} 70 \mathrm{~S} 6 \mathrm{~K}$ signaling pathway. Tumour Biol 2014;35:10707-10714.

20. Hait NC, Wise LE, Allegood JC, et al. Active, phosphorylated fingolimod inhibits histone deacetylases and facilitates fear extinction memory. Nat Neurosci 2014;17:971-980.

21. di Nuzzo L, Orlando R, Tognoli C, et al. Antidepressant activity of fingolimod in mice. Pharmacol Res Perspect 2015;3:e0135.

22. Hemmati F, Dargahi L, Nasoohi S, et al. Neurorestorative effect of FTY720 in a rat model of Alzheimer's disease: comparison with memantine. Behav Brain Res 2013;252:415-421.

23. Gao F, Liu Y, Li X, Wang Y, Wei D, Jiang W. Fingolimod (FTY720) inhibits neuroinflammation and attenuates spontaneous convulsions in lithium-pilocarpine induced status epilepticus in rat model. Pharmacol Biochem Behav 2012;103:187-196.

24. Gol M, Ghorbanian D, Hassanzadeh S, Javan M, Mirnajafi-Zadeh J, Ghasemi-Kasman M. Fingolimod enhances myelin repair of hippocampus in pentylenetetrazol-induced kindling model. Eur J Pharm Sci 2017;96:72-83.

25. Jafarian M, Karimzadeh F, Alipour F, et al. Cognitive impairments and neuronal injury in different brain regions of a genetic rat model of absence epilepsy. Neuroscience 2015;298:161-170.

26. Sarkisova K, van Luijtelaar G. The WAG/Rij strain: a genetic animal model of absence epilepsy with comorbidity of depression [corrected]. Prog Neuropsychopharmacol Biol Psychiatry 2011;35:854-876

27. Russo E, Citraro R, Constanti A, et al. Upholding WAG/Rij rats as a model of absence epileptogenesis: hidden mechanisms and a new theory on seizure development. Neurosci Biobehav Rev 2016;71: 388-408.

28. Van Luijtelaar G, Lyashenko S, Vastyanov R, et al. Cytokines and absence seizures in a genetic rat model. Neurophysiology 2012;43: 478-486.

29. Russo E, Andreozzi F, Iuliano R, et al. Early molecular and behavioral response to lipopolysaccharide in the WAG/Rij rat model of absence epilepsy and depressive-like behavior, involves interplay between AMPK, AKT/mTOR pathways and neuroinflammatory cytokine release. Brain Behav Immun 2014;42:157-168.

30. Kovacs Z, Dobolyi A, Juhasz G, Kekesi KA. Lipopolysaccharide induced increase in seizure activity in two animal models of absence epilepsy WAG/Rij and GAERS rats and Long Evans rats. Brain Res Bull 2014;104:7-18.

31. Russo E, Citraro R, Donato G, et al. mTOR inhibition modulates epileptogenesis, seizures and depressive behavior in a genetic rat model of absence epilepsy. Neuropharmacology 2013;69:25-36.

32. Balosso S, Ravizza T, Aronica E, Vezzani A. The dual role of TNFalpha and its receptors in seizures. Exp Neurol 2013;247:267-271.

33. Citraro R, Leo A, Constanti A, Russo E, De Sarro G. mTOR pathway inhibition as a new therapeutic strategy in epilepsy and epileptogenesis. Pharmacol Res 2016;107:333-343.

34. Jagirdar R, Drexel M, Kirchmair E, Tasan RO, Sperk G. Rapid changes in expression of class I and IV histone deacetylases during epileptogenesis in mouse models of temporal lobe epilepsy. Exp Neurol 2015;273:92-104.

35. Leo A, Constanti A, Coppola A, Citraro R, De Sarro G, Russo E. mTOR signaling in epilepsy and epileptogenesis: preclinical and clinical studies. In: Maiese K, editor. Molecules to medicine with mTOR: translating critical pathways into novel therapeutic strategies Elsevier Inc.; 2016. p. 123-142.

36. Citraro R, Leo A, Aiello R, Pugliese M, Russo E, De Sarro G. Comparative analysis of the treatment of chronic antipsychotic 
drugs on epileptic susceptibility in genetically epilepsy-prone rats. Neurotherapeutics 2015;12:250-262.

37. Kuznetsova GD, Petrova EV, Coenen AM, Van Luijtelaar EL. Generalized absence epilepsy and catalepsy in rats. Physiol Behav 1996;60:1165-1169.

38. Midzyanovskaya IS, Shatskova AB, Sarkisova KY, van Luijtelaar G, Tuomisto L, Kuznetsova GD. Convulsive and nonconvulsive epilepsy in rats: effects on behavioral response to novelty stress. Epilepsy Behav 2005;6:543-651.

39. Citraro R, Leo A, Franco V, et al. Perampanel effects in the WAG/ Rij rat model of epileptogenesis, absence epilepsy, and comorbid depressive-like behavior. Epilepsia 2017;58:231-238.

40. Russo E, Citraro R, Scicchitano F, et al. Effects of early long-term treatment with antiepileptic drugs on development of seizures and depressive-like behavior in a rat genetic absence epilepsy model. Epilepsia 2011;52:1341-1350.

41. Citraro R, Leo A, De Fazio P, De Sarro G, Russo E. Antidepressants but not antipsychotics have antiepileptogenic effects with limited effects on comorbid depressive-like behaviour in the WAG/Rij rat model of absence epilepsy. Br J Pharmacol 2015;172:3177-3188.

42. Russo E, Citraro R, Scicchitano F, et al. Vigabatrin has antiepileptogenic and antidepressant effects in an animal model of epilepsy and depression comorbidity. Behav Brain Res 2011;225:373-376.

43. Russo E, Constanti A, Ferreri G, Citraro R, De Sarro G. Nifedipine affects the anticonvulsant activity of topiramate in various animal models of epilepsy. Neuropharmacology 2004;46:865-878.

44. Meno-Tetang GM, Li H, Mis S, et al. Physiologically based pharmacokinetic modeling of FTY720 (2-amino-2[2-(-4octylphenyl)ethyl]propane-1,3-diol hydrochloride) in rats after oral and intravenous doses. Drug Metab Dispos 2006;34:1480-1487.

45. Blumenfeld H, Klein JP, Schridde U, et al. Early treatment suppresses the development of spike-wave epilepsy in a rat model. Epilepsia 2008;49:400-409.

46. Bogdanova OV, Kanekar S, D'Anci KE, Renshaw PF. Factors influencing behavior in the forced swim test. Physiol Behav 2013;118:227-239.

47. Russo E, Chimirri S, Aiello R, et al. Lamotrigine positively affects the development of psychiatric comorbidity in epileptic animals, while psychiatric comorbidity aggravates seizures. Epilepsy Behav 2013;28:232-240.

48. Citraro R, Gallelli L, Leo A, et al. Effects of chronic sodium alendronate on depression and anxiety in a menopausal experimental model. Pharmacol Biochem Behav 2015;129:65-71.

49. Tsuji M, Takeda H, Matsumiya T. Modulation of passive avoidance in mice by the 5-HT1A receptor agonist flesinoxan: comparison with the benzodiazepine receptor agonist diazepam. Neuropsychopharmacology 2003;28:664-674.

50. Nestler EJ, Hyman SE. Animal models of neuropsychiatric disorders. Nat Neurosci 2010;13:1161-1169.

51. Sarkisova KY, Kulikov MA. Behavioral characteristics of WAG/Rij rats susceptible and non-susceptible to audiogenic seizures. Behav Brain Res 2006;166:9-18.

52. Zovkic IB, Sweatt JD. Epigenetic mechanisms in learned fear: implications for PTSD. Neuropsychopharmacology 2013;38:77-93.

53. Sakurai M, Sekiguchi M, Zushida K, et al. Reduction in memory in passive avoidance learning, exploratory behaviour and synaptic plasticity in mice with a spontaneous deletion in the ubiquitin Cterminal hydrolase L1 gene. Eur J Neurosci 2008;27:691-701.

54. Karson A, Utkan T, Balci F, Aricioglu F, Ates N. Age-dependent decline in learning and memory performances of WAG/Rij rat model of absence epilepsy. Behav Brain Funct 2012;8:51.

55. Monville C, Torres EM, Dunnett SB. Comparison of incremental and accelerating protocols of the rotarod test for the assessment of motor deficits in the 6-OHDA model. J Neurosci Meth 2006;158: 219-223.
56. Park HW, Chang JW, Yang YS, et al. The effect of donor-dependent administration of human umbilical cord blood-derived mesenchymal stem cells following focal cerebral ischemia in rats. Exp Neurobiol 2015;24:358-365.

57. Amodio N, Stamato MA, Gulla AM, et al. Therapeutic targeting of miR-29b/HDAC4 epigenetic loop in multiple myeloma. Mol Cancer Ther 2016;15:1364-1375.

58. Gouveia TL, Vieira de Sousa PV, de Almeida SS, et al. High serum levels of proinflammatory markers during epileptogenesis. Can omega-3 fatty acid administration reduce this process? Epilepsy Behav 2015;51:300-305.

59. Vezzani A, Aronica E, Mazarati A, Pittman QJ. Epilepsy and brain inflammation. Exp Neurol 2013;244:11-21.

60. de Vries EE, van den Munckhof B, Braun KP, van Royen-Kerkhof A, de Jager W, Jansen FE. Inflammatory mediators in human epilepsy: a systematic review and meta-analysis. Neurosci Biobehav Rev 2016;63:177-190.

61. van Luijtelaar G, Zobeiri M. Progress and outlooks in a genetic absence epilepsy model (WAG/Rij). Curr Med Chem 2014;21: 704-721.

62. Kovacs Z, Czurko A, Kekesi KA, Juhasz G. Intracerebroventricularly administered lipopolysaccharide enhances spike-wave discharges in freely moving WAG/Rij rats. Brain Res Bull 2011;85:410-416.

63. Kovacs Z, Kekesi KA, Szilagyi N, et al. Facilitation of spike-wave discharge activity by lipopolysaccharides in Wistar Albino Glaxo/ Rijswijk rats. Neuroscience 2006;140:731-742.

64. Rimoli MG, Russo E, Cataldi M, et al. T-type channel blocking properties and antiabsence activity of two imidazo[1,2-b]pyridazine derivatives structurally related to indomethacin. Neuropharmacology 2009;56:637-646.

65. Akin D, Ravizza T, Maroso M, et al. IL-1beta is induced in reactive astrocytes in the somatosensory cortex of rats with genetic absence epilepsy at the onset of spike-and-wave discharges, and contributes to their occurrence. Neurobiol Dis 2011;44:259-269.

66. Russo E, Follesa P, Citraro R, et al. The mTOR signaling pathway and neuronal stem/progenitor cell proliferation in the hippocampus are altered during the development of absence epilepsy in a genetic animal model. Neurol Sci 2014;35:1793-1799.

67. Zhang B, Wong M. Pentylenetetrazole-induced seizures cause acute, but not chronic, mTOR pathway activation in rat. Epilepsia 2012;53:506-511.

68. Song GY, Kang JS, Lee SY, Myung CS. Region-specific reduction of Gbeta4 expression and induction of the phosphorylation of PKB/ Akt and ERK1/2 by aging in rat brain. Pharmacol Res 2007;56:295302.

69. Orellana AM, Vasconcelos AR, Leite JA, et al. Age-related neuroinflammation and changes in AKT-GSK-3beta and WNT/betaCATENIN signaling in rat hippocampus. Aging (Albany NY) 2015;7:1094-1111.

70. Sankar R, Mazarati A. Neurobiology of depression as a comorbidity of epilepsy. In: Noebels JL, Avoli M, Rogawski MA, Olsen RW, Delgado-Escueta AV, editors. Jasper's basic mechanisms of the epilepsies, Bethesda (MD), 2012.

71. Kanner AM, Mazarati A, Koepp M. Biomarkers of epileptogenesis: psychiatric comorbidities (?). Neurotherapeutics 2014;11:358-72.

72. Sarkisova KY, Kuznetsova GD, Kulikov MA, van Luijtelaar G. Spike-wave discharges are necessary for the expression of behavioral depression-like symptoms. Epilepsia 2010;51:146-160.

73. Fox E, Edwards K, Burch G, et al. Outcomes of switching directly to oral fingolimod from injectable therapies: Results of the randomized, open-label, multicenter, Evaluate Patient OutComes (EPOC) study in relapsing multiple sclerosis. Mult Scler Relat Disord 2014;3:607-619.

74. Hunter SF, Agius M, Miller DM, et al. Impact of a switch to fingolimod on depressive symptoms in patients with relapsing 
multiple sclerosis: an analysis from the EPOC (Evaluate Patient OutComes) trial. J Neurol Sci 2016;365:190-198.

75. Bang SR, Ambavade SD, Jagdale PG, Adkar PP, Waghmare AB, Ambavade PD. Lacosamide reduces HDAC levels in the brain and improves memory: Potential for treatment of Alzheimer's disease. Pharmacol Biochem Behav 2015;134:65-69.

76. Nalivaeva NN, Belyaev ND, Lewis DI, et al. Effect of sodium valproate administration on brain neprilysin expression and memory in rats. J Mol Neurosci 2012;46:569-577.

77. Kilgore M, Miller CA, Fass DM, et al. Inhibitors of class 1 histone deacetylases reverse contextual memory deficits in a mouse model of Alzheimer's disease. Neuropsychopharmacology 2010;35:870-880.

78. Sitnikova E, Rutskova EM, Raevsky VV. Reduction of epileptic spike-wave activity in WAG/Rij rats fostered by Wistar dams. Brain Res 2015;1594:305-309.

79. Sitnikova E. Neonatal sensory deprivation promotes development of absence seizures in adult rats with genetic predisposition to epilepsy. Brain Res 2011;1377:109-118.

80. Deogracias R, Yazdani M, Dekkers MP, et al. Fingolimod, a sphingosine-1 phosphate receptor modulator, increases BDNF levels and improves symptoms of a mouse model of Rett syndrome. Proc Natl Acad Sci U S A 2012;109:14230-14235.

81. Calfa G, Chapleau CA, Campbell S, et al. HDAC activity is required for BDNF to increase quantal neurotransmitter release and dendritic spine density in CA1 pyramidal neurons. Hippocampus 2012;22:1493-1500.

82. Palleria C, Leporini C, Maida F, et al. Potential effects of current drug therapies on cognitive impairment in patients with type 2 diabetes. Front Neuroendocrinol. 2016;42:76-92.

83. Jagirdar R, Drexel M, Bukovac A, Tasan RO, Sperk G. Expression of class II HDACs in two mouse models of temporal lobe epilepsy. J Neurochem 2015 Nov 25 [Epub ahead of print].

84. Morris MJ, Karra AS, Monteggia LM. Histone deacetylases govern cellular mechanisms underlying behavioral and synaptic plasticity in the developing and adult brain. Behav Pharmacol 2010;21:409419.

85. French JA, White HS, Klitgaard H, et al. Development of new treatment approaches for epilepsy: unmet needs and opportunities. Epilepsia 2013;54(Suppl. 4):3-12. 